\title{
Myelination of preterm brain networks at adolescence
}

\author{
Beatriz Laureano ${ }^{1,4}$, Hassna Irzan ${ }^{2,1}$, Sebastien Ourselin ${ }^{1,2}$, Neil Marlow $^{3}$, and \\ Andrew Melbourne ${ }^{1,2}$ \\ ${ }^{1}$ School of Biomedical Engineering \& Imaging Sciences, King's College London, UK. \\ ${ }^{2}$ Dept. of Medical Physics and Biomedical Engineering, University College London, \\ London, UK. ${ }^{3}$ Institute for Women's Health, University College London, London, \\ UK. ${ }^{4}$ beatriz.laureano.tic@gmail.com
}

\begin{abstract}
Prematurity and preterm stressors severely affect the development of infants born before 37 weeks of gestation, with increasing effects seen at earlier gestations. Although preterm mortality rates have declined due to the advances in neonatal care, disability rates, especially in middle-income settings, continue to grow. With the advances in MRI imaging technology, there has been a focus on safely imaging the preterm brain to better understand its development and discover the brain regions and networks affected by prematurity. Such studies aim to support interventions and improve the neurodevelopment of preterm infants and deliver accurate prognoses. Few studies, however, have focused on the fully developed brain of preterm born infants, especially in extremely preterm subjects. To assess the long-term effect of prematurity on the adult brain, myelin related biomarkers such as myelin water fraction and g-ratio are measured for a cohort of 19-year-old extremely preterm subjects. Using multi-modal imaging techniques that combine T2 relaxometry and neurite density information, the results show that specific regions of the brain associated with white matter injuries due to preterm birth, such as the Posterior Limb of the Internal Capsule and Corpus Callosum, are still less myelinated in adulthood. Such findings might imply reduced connectivity in the adult preterm brain and explain the poor cognitive outcome.
\end{abstract}

\section{Introduction}

Prematurity is the leading cause of death in children under the age of five, with preterm birth rates continuing to increase in almost every country with reliable data [14]. Despite the medical innovations in prenatal care, extremely preterm (EPT) infants (born before 26 weeks of gestation) remain at a high risk of death (30\%-50\% mortality). In addition, despite improved survival, disability rates are not declining, specifically in middle-income settings[14,5]. Preterm birth and other perinatal stressors such as premature exposure to the extrauterine environment, ischemia, hypoxia, and inflammation can lead to White Matter (WM) injuries or otherwise affect brain development; more drastically, these can subsequently result in hypomyelination and long-term alterations of 
the brain's connectivity and structural complexity [17]. Therefore, measurement of myelin density and the spatial variation of myelin has been used to assess brain maturation in EPT babies and as a predictor of neurodevelopmental outcome, demonstrating the link between preterm brain development and significant alterations which can relate to cognitive performance [4,17]. Few studies have focused on the fully developed brain of preterm born individuals, where myelination processes are developed [11]. This study attempts to overcome this lack of knowledge by examining a cohort of 19-year-old participants, born at both extremely preterm and term gestation, and combining the structural sensitivity, but myelin non-specificity, of DWI and the high myelin specificity, but structural insensitivity of T2 relaxometry to reveal changes in myelin related biomarkers. First, full brain analysis is performed, followed by a region-specific approach investigating areas hypothesised to be more affected by preterm stressors such as the Corpus Callosum and the Posterior Limb of the Internal Capsule (PLIC). Lastly, these biomarkers were analysed between defined cortical regions and along the pathways between regions for a more thorough look at functional connectivity, and hypomyelination [10]. Figure 1 report the main steps of the analysis.

\section{Methods}

\subsection{Data}

Imaging data were acquired for a cohort of 142 adolescents at 19 years of age. Data for 89 EPT adolescents (52 Female/37 Male) and 53 term-born socioeconomically matched peers (32 Female/22 Male) were acquired on a $3 \mathrm{~T}$ Phillips Achieva. Diffusion weighted data (DWI) was acquired across four b-values at $\mathrm{b}$ $=0,300,700,2000 \mathrm{smm}^{-2}$ with $\mathrm{n}=4,8,16,32$ directions respectively at $\mathrm{TE}=70 \mathrm{~ms}$ $(2.5 \times 2.5 \times 3.0 \mathrm{~mm})$. T2 weighted data was acquired in the same space as the diffusion imaging with ten echo times at $\mathrm{TE}=13,16,19,25,30,40,50,85,100,150 \mathrm{~ms}$ $(2.5 \times 2.5 \times 3.0 m m)$. In addition we acquired 3D T1-weighted $(\mathrm{TR} / \mathrm{TE}=6.93 / 3.14 \mathrm{~ms})$ volume at $1 \mathrm{~mm}$ isotropic resolution for segmentation and parcellation [3]. B0 field maps were acquired to correct for EPI-based distortions between the diffusion imaging and the T1-weighted volumes. All participants gave informed consent before taking part in the experiment. Ethical approval was granted by the South Central - Hampshire Research Ethics Committee.

\subsection{Region of interest values}

Based upon previous research in these areas, manual regions of interest were described in the genu and splenium of the corpus callosum in addition to the posterior limb of the internal capsule $[13,1]$. These regions are ordinarily highlymyelinated white matter regions providing communication between cerebral hemispheres and carrying signals relating to motor-function to the rest of the body. 


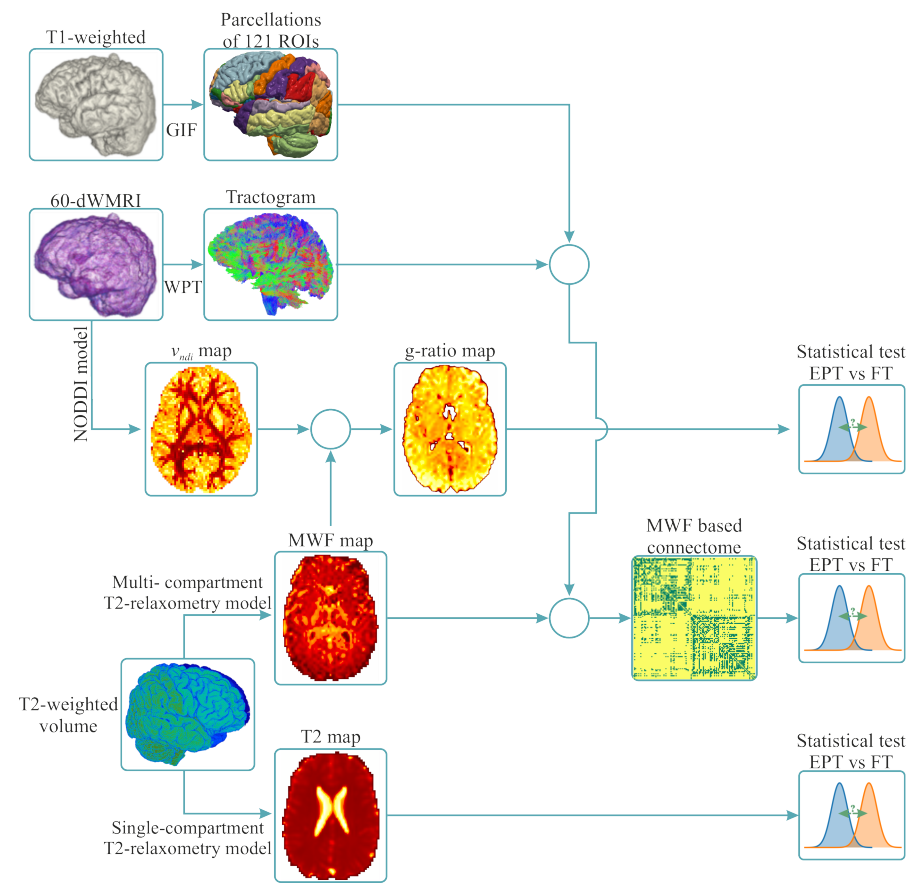

Fig. 1. Overview of methodology pipeline: T2-maps are estimated from the single compartment T2-relaxometry model, Myelin Water Fraction (MWF) maps are measured from the multi-compartment T2-relaxometry model. Neurite Density Index from the NODDI model and MWF maps are used to compute g-ratio maps (Eq. 2). A tractogram is generated by performing Whole-brain Probabilistic Tractography (WPT) for each subject from the diffusion-weighted data (DWI). Combining the streamline information with the maps of MWF, brain connectomes are derived for each subject. Statistical tests are performed to detect differences in T2, g-ratio, and MWF-derived markers between FT and EPT subjects.

\subsection{Model Fitting}

Myelin Water Fraction (MWF) corresponds to the volume of myelin water in a determined area of the brain and it is a surrogate measure for myelin content. Brain water T2 decreases with increasing gestational and postnatal age and is a more sensitive marker of brain maturation than visual assessment of $\mathrm{T} 2$-weighted MR images [9].

Ordinarily a single $\mathrm{T} 2$ value can be fitted to multi-echo data. This assumes that each voxel of the brain image contains a single biological compartment. Brain modelling can be enhanced by assuming that each brain voxel contains multiple compartments with different corresponding T2 values [12]. In the absence of a significant fluid component, brain tissue can be described with two compartments, one of myelin water with short $\mathrm{T} 2$ and one of other intra and 
extra-cellular space with significantly longer T2. The volume fraction of the MWF component $\left(v_{m w f}\right)$ is thus a marker of myelin density (Eq. 1).

$$
S(T E)=S_{0}\left[v_{m w f} e^{-\frac{T E}{T 2_{1}}}+\left(1-v_{m w f}\right) e^{-\frac{T E}{T 2_{2}}}\right]
$$

DWI is sensitive to local structure, but due to the short T2 of the myelin signal, DWI is non-specific to myelin content. The g-ratio is a geometrical invariant of axons quantifying their degree of myelination relative to their cross-sectional size. This biomarker can be calculated from MWF $\left(v_{m w f}\right)$ and NDI $\left(v_{n d i}\right)$ data when assuming a cylindrical geometry of the axons since these are both density related biomarkers (Eq. 2) [12]. Signal conduction velocity can be shown to be proportional to $\gamma \sqrt{-\log \gamma}[11]$

$$
\gamma=\left(\frac{v_{m w f}}{v_{n d i}}+1\right)^{-\frac{1}{2}}
$$

\subsection{Brain Network and Feature Extraction}

A Multi-Shell Multi-Tissue (MSTMT) approach was employed to estimate the response function for White Matter (WM), Grey Matter (GM), and Cerebrospinal fluid (CSF) [8]. In addition, Constrained Spherical Deconvolution (CSD) is utilized to evaluate the orientation distribution of the WM fibres [16]. Ten million streamlines are estimated for each subject by performing whole-brain probabilistic tractography [15]. Tissue parcellations are obtained by applying the Geodesic Information Flow labelling protocol to the T1-weighted volumes [3]. Neurite Density Index $\left(v_{n d i}\right)$ is estimated with the Neurite Orientation Dispersion and Density Imaging (NODDI) model [18]. One hundred twenty-one GM regions (ROIs) formed the nodes of the brain network. For each subject, a graph $\mathcal{G}=(V, E)$ is defined, where the nodes $V$ are the ROI, and $E$ corresponds to the set of edges connecting the ROIs. In $\mathcal{G}$, along each streamline connecting nodes $i$ and $j$, the strength of connectivity of the edge $(i, j)$ is defined as the mean sampled value of MWF. A more detailed description is provided by a previous study [7].

\section{Results}

\subsection{Overall brain}

Single-compartment T2 maps of the entire cohort; as well as MWF and g-ratio maps were generated and examples are shown in figure 2 . There was no significant difference for MWF and g-ratio values between EPT and FT participants when analysing the whole brain but there was a significant difference for $\mathrm{T} 2$ values which were higher in the EPT cohort (p-value 0.00137). The T2, MWF and gratio averages of the whole brain for EPT and FT participants were (90.85 \pm 9.00 , $86.40 \pm 4.23) ;(0.23 \pm 0.03,0.23 \pm 0.03)$ and $(0.84 \pm 0.02,0.85 \pm 0.02)$ respectively. Figure 3 shows the correlation between g-ratio/MWF (p-value $7.3^{-34}$ and $\rho$ 0.82 ) and g-ratio/NDI (p-value $6.7^{-5}$ and $\rho$ 0.34); with g-ratio/MWF exhibiting a more significant correlation. 


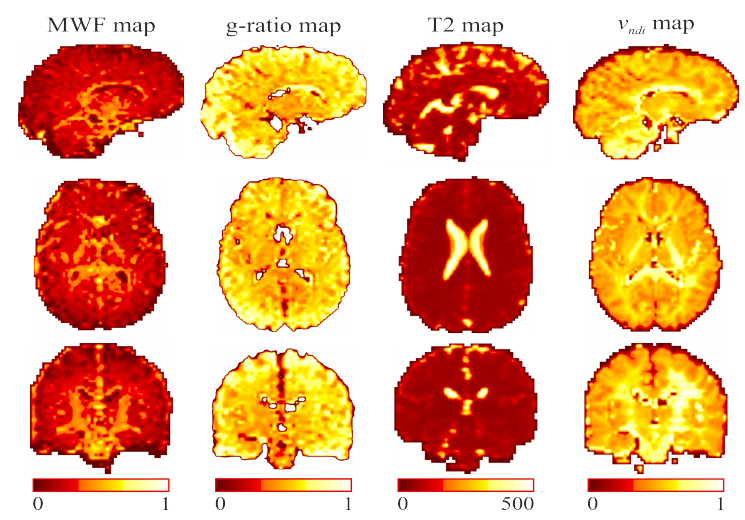

Fig. 2. Example of Myelin Water Fractio (MWF), g-ratio, T2, and Neurite Density Index $\left(v_{n d i}\right)$ maps of an EPT participant.

\subsection{Corpus Callosum and Posterior Limb of Internal Capsule:}

The Corpus Callosum was analysed and figure 4 shows an average reduction of myelin in EPT born participants $(0.31 \pm 0.06)$ when compared to FT $(0.34 \pm 0.05)$; overall (p-value $1.6^{-3}$ ). It is worth highlighting that the Splenium of the Corpus Callosum showed the highest significant difference in the corpus callosum (higher than the genu ), for EPT $(0.31 \pm 0.06)$, and FT $(0.35 \pm 0.050)$, with (p-value $\left.1.92 e^{-5}\right)$. The PLIC region was analysed using non-parametric approaches and figure 5 shows a significant lower MWF value in EPT born participants (p-value 0.026). The thalamus was also analysed but showed no difference between EPT and FT born participants.

For the corpus callosum the results of g-ratio measurement show a statistically significant difference (p-value 0.0266 ) between EPT and FT participants; with g-ratio of EPT being higher $(0.830 \pm 0.031)$ than FT $(0.819 \pm$ 0.0254).Furthermore, g-ratio results in the Splenium of the Corpus Callosum presented a more significant difference (p-value 0.0015) between EPT $(0.834 \pm$ $0.0341)$ and FT $(0.816 \pm 0.026)$ participants. A difference in conduction velocity (p-value 0.022) was also found for the corpus callosum with EPT participants' values being lower $(0.355 \pm 0.0245)$ than FT $(0.364 \pm 0.0180)$; and specifically for the splenium of the corpus callosum (p-value 0.0023$)$ where EPT $(0.352 \pm 0.030)$ and FT $(0.366 \pm 0.017)$. These results alongside the g-ratio ones are depicted in figure 6. No significant statistical difference was found for PLIC.

\subsection{Region connectivity and specific pathways:}

Decreased MWF was observed in the R.Thalamus-L.Calcarine Cortex (p-value= 0.03 ) and in the R.Thalamus- L.Precuneus ( $\mathrm{p}$-value $=0.018$ ) pathways in EPT born participants. Parietal-Frontal pathways were also analysed but no significant difference was registered between EPT and FT participants. The MWF 

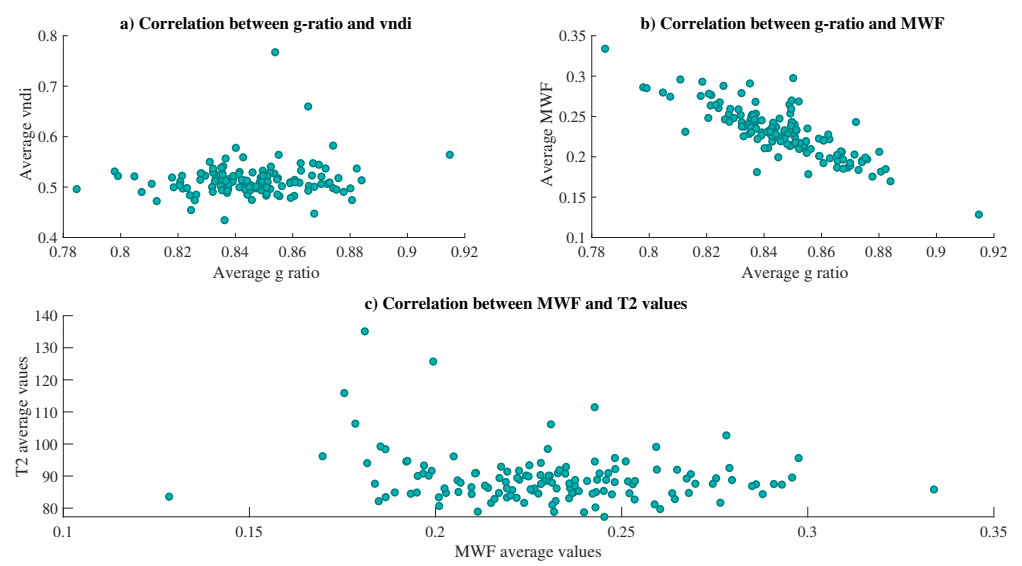

Fig. 3. Correlation plots a) $v_{n d i} /$ g-ratio b) $\mathrm{MWF} /$ g-ratio c) $\mathrm{MWF} / \mathrm{T} 2$.

connectomes were analysed and the average MWF of all myelin containing networks was found to be $(0.240 \pm 0.037)$ and $(0.244 \pm 0.036)$ respectively for EPT and FT born participants. No significant difference was found for these groups. Using the same approach for all networks within one region; all 121 regions were analysed and multiple regions appeared to have a decreased overall myelin density; table 1 highlights the regions where a significant difference in MWF values was found and the corresponding p-values and CIs.

\section{Discussion}

The average MWF values of the whole brain showed no significant differences between EPT and FT participants. This might suggest that the effect of preterm birth is localised to specific brain regions, resulting in some brain areas being more affected. The preterm brain, having to develop exvivo, will reorganise its structure and develop differently in comparison to infants still in the womb, which is an indication that EPT born infants might present tracts with different characteristics than those in FT born individuals to compensate for the different developmental conditions $[6,7]$.

Injuries in the Corpus Callosum are considerably frequent in prematurity due to being adjacent to the periventricular brain region, which is often impaired. In addition, underdevelopment can lead to disruption of intra-hemispheric communication and reduce connectivity [13]. The findings in the Corpus Callosum and specifically in the Splenium, which appears less myelinated in EPT than FT born participants, indicate the persistence of preterm birth consequences into adolescence.

The low MWF results found for the PLIC in EPT born participants might be an indicator of motor pathway underdevelopment or disruption of myelination 

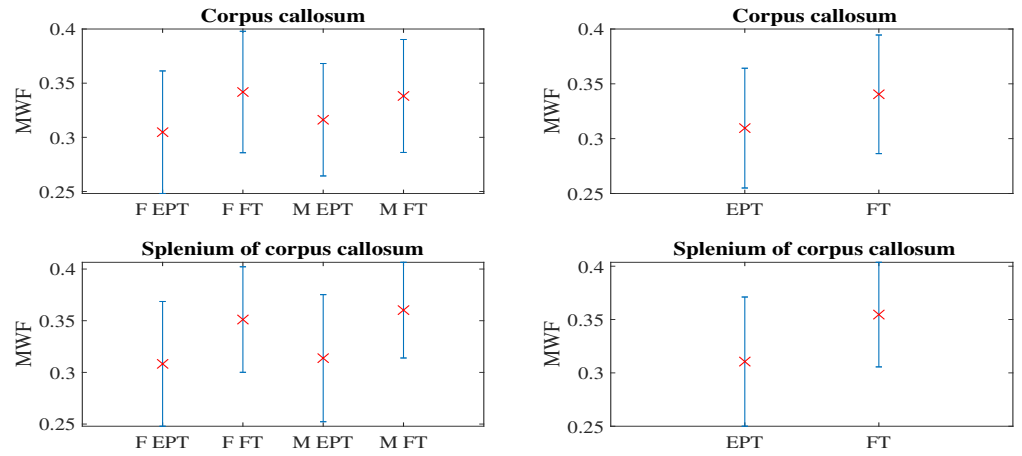

Fig. 4. Differences in the Corpus Callosum and the Splenium of the Corpus Callosum MWF information for preterm, term, female and male participants showed in 4 error bars.
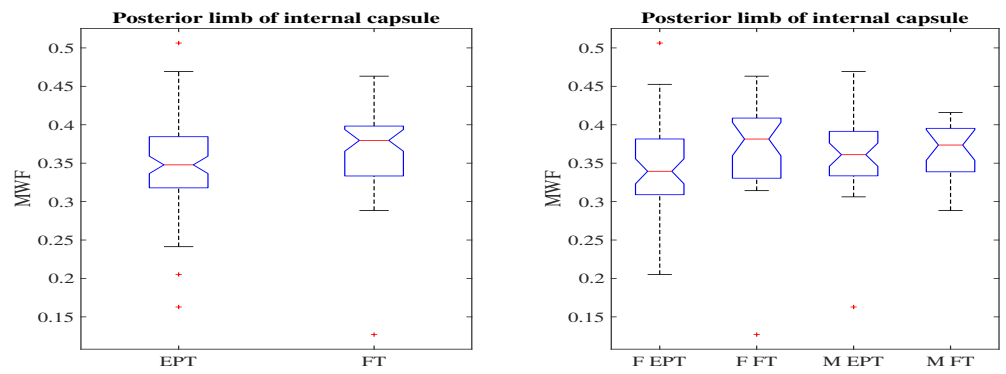

Fig. 5. Differences in the PLIC MWF information for EPT, FT, female and male participants showed in 2 boxplot graphs.
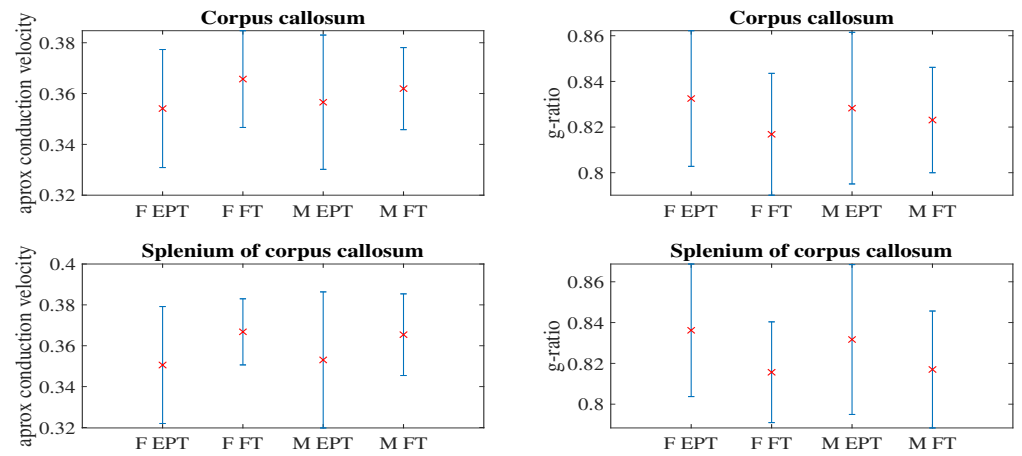

Fig. 6. g-ratio and conduction velocity error bars for the Corpus Callosum and Splenium of Corpus Callosum for all groups. 


\begin{tabular}{|c|c|c|c|c|}
\hline $\begin{array}{c}\text { Regions where MWF } \\
\text { EPT < FT }\end{array}$ & $\begin{array}{c}\text { EPT MWF } \\
\text { mean (std) }\end{array}$ & $\begin{array}{c}\text { FT MWF } \\
\text { mean (std) }\end{array}$ & p-value & 95 C.I \\
\hline Left Superior Occipital Gyrus & $0.23(0.041)$ & $0.24(0.040)$ & 0.026 & $-0.031-0.0020$ \\
\hline Left Calcarine Cortex & $0.23(0.042)$ & $0.25(0.037)$ & $\mathbf{0 . 0 0 8 4}$ & $-0.034-0.0051$ \\
\hline Left Cuneus & $0.22(0.042)$ & $0.24(0.039)$ & 0.0101 & $-0.034-0.0046$ \\
\hline Left Occipital pole & $0.22(0.039)$ & $0.23(0.039)$ & 0.029 & $-0.029-0.0016$ \\
\hline Right Middle Temporal Gyrus & $0.23(0.036)$ & $0.24(0.033)$ & 0.025 & $-0.027-0.0018$ \\
\hline Right Fusiform Gyrus & $0.22(0.034)$ & $0.24(0.033)$ & $\mathbf{0 . 0 0 1 0}$ & $-0.032-0.0082$ \\
\hline R Inferior Temporal Gyrus & $0.22(0.036)$ & $0.24(0.032)$ & $\mathbf{0 . 0 0 2 0}$ & $-0.032-0.0073$ \\
\hline R superior parietal lobe & $0.22(0.038)$ & $0.23(0.038)$ & 0.035 & $-0.028-0.00099$ \\
\hline Right Angular Gyrus & $0.22(0.036)$ & $0.23(0.034)$ & 0.0438 & $-0.026-0.00037$ \\
\hline Right Precuneus & $0.23(0.040)$ & $0.24(0.037)$ & 0.0481 & $-0.028-0.00012$ \\
\hline Right Superior occipital Gyrus & $0.20(0.037)$ & $0.23(0.033)$ & $\mathbf{0 . 0 0 0 5}$ & $-0.035-0.010$ \\
\hline Right Calcarine Cortex & $0.21(0.040)$ & $0.24(0.035)$ & $\mathbf{0 . 0 0 1 0}$ & $-0.037-0.0095$ \\
\hline Right Cuneus & $0.20(0.038)$ & $0.22(0.034)$ & 0.0043 & $-0.032-0.0061$ \\
\hline Right Occipital pole & $0.19(0.043)$ & $0.21(0.036)$ & $\mathbf{0 . 0 0 1 9 2}$ & $-0.037-0.0086$ \\
\hline Right lingual Gyrus & $0.22(0.038)$ & $0.24(0.038)$ & $\mathbf{0 . 0 0 0 8 4}$ & $-0.037-0.0099$ \\
\hline Right Occipital Fusiform Gyrus & $0.19(0.037)$ & $0.22(0.032)$ & $\mathbf{0 . 0 0 0 2 7}$ & $-0.0359-0.0110$ \\
\hline Right Inferior occipital Gyrus & $0.20(0.036)$ & $0.22(0.031)$ & $\mathbf{0 . 0 0 0 5}$ & $-0.034-0.0097$ \\
\hline Right Middle occipital Gyrus & $0.21(0.039)$ & $0.22(0.034)$ & 0.030 & $-0.0279-0.00143$ \\
\hline
\end{tabular}

Table 1. Table showing the regions of the brain where overall MWF are lower in EPT participants. The p-values, Confidence Intervals (C.I), and means with standard deviation (std) are reported. The results that survived the Bonferroni threshold of $0.05 / 18=0.0028$ are in bold. 
processes due to preterm stressors, which have led to the hypomyelination of these pathways and possible motor deficits extended into adolescence [17].

The decrease in myelin-linked markers in the Calcarine cortex, which is the main site of input signals coming from the retina, alongside the Precuneus, Cuneus and Lingual Gyrus, indicates differences in the development of brain cortex and possibly visual deficits. This might be caused by extra-uterine development which led to reduced functional connectivity. Another option is that compensatory mechanisms led to the formation of additional pathways going from those regions which are hypomyelinated and therefore not entirely functional. This hypothesis could be tested in future work. In addition, the networks from the Precuneus and the Calcarine cortex to the thalamus also presented lower MWF values or efficient connectivity, thus demonstrating the effects of the disruption of myelination of the thalamocortical radiations [2].

The regions and pathways that we have found to be significantly affected by preterm birth are related to cognitive functions such as speech, sight and memory. Therefore, lower myelination in these areas could potentially represent the result of injuries suffered after prematurity, leading to hypomyelination and thus to functional deficits that propagated through adolescence, continuing to affect individuals born extremely prematurely.

No significant difference in results between female and male participants was registered; however, most results remained significant when distinguishing between sexes. Some regions, however, did not show a significant difference in EPT v FT individuals in the male cohort, but this might be due to the smaller sample size. In conclusion, our results support the hypothesis that preterm stressors affect brain maturation leading to changes in overall brain networks and in specific brain areas.

Acknowledgements This work is supported by the EPSRC-funded UCL Centre for Doctoral Training in Medical Imaging (EP/L016478/1), the Department of Health NIHR-funded Biomedical Research Centre at University College London Hospitals and Medical Research Council (MR/N024869/1).

\section{References}

1. Longitudinal study of white matter development and outcomes in children born very preterm. Cerebral Cortex, 27(8):4094-4105, aug 2017.

2. Gareth Ball, James P Boardman, Paul Aljabar, Anand Pandit, Tomoki Arichi, Nazakat Merchant, Daniel Rueckert, A David Edwards, and Serena J Counsell. The influence of preterm birth on the developing thalamocortical connectome. Cortex, 49(6):1711-1721, 2013.

3. M Jorge Cardoso, Marc Modat, Robin Wolz, Andrew Melbourne, David Cash, Daniel Rueckert, and Sebastien Ourselin. Geodesic information flows: spatiallyvariant graphs and their application to segmentation and fusion. IEEE transactions on medical imaging, 34(9):1976-1988, 2015.

4. Nicholas Dingwall, Alan Chalk, Teresa I Martin, Catherine J Scott, Carla Semedo, Quan Le, Eliza Orasanu, Jorge M Cardoso, Andrew Melbourne, Neil Marlow, et al. 
T2 relaxometry in the extremely-preterm brain at adolescence. Magnetic resonance imaging, 34(4):508-514, 2016.

5. Hannah C Glass, Andrew T Costarino, Stephen A Stayer, Claire Brett, Franklyn Cladis, and Peter J Davis. Outcomes for extremely premature infants. Anesthesia and analgesia, 120(6):1337, 2015.

6. Elveda Gozdas, Nehal A Parikh, Stephanie L Merhar, Jean A Tkach, Lili He, and Scott K Holland. Altered functional network connectivity in preterm infants: antecedents of cognitive and motor impairments? Brain Structure and Function, 223(8):3665-3680, 2018.

7. Hassna Irzan, Erika Molteni, Michael Hütel, Sebastien Ourselin, Neil Marlow, and Andrew Melbourne. White matter analysis of the extremely preterm born adult brain. NeuroImage, 237:118112, 2021.

8. Ben Jeurissen, Jacques-Donald Tournier, Thijs Dhollander, Alan Connelly, and Jan Sijbers. Multi-tissue constrained spherical deconvolution for improved analysis of multi-shell diffusion mri data. NeuroImage, 103:411-426, 2014.

9. Alex MacKay, Cornelia Laule, Irene Vavasour, Thorarin Bjarnason, Shannon Kolind, and Burkhard Mädler. Insights into brain microstructure from the T2 distribution, 2006.

10. Atul Malhotra, Michael Ditchfield, Michael C Fahey, Margie Castillo-Melendez, Beth J Allison, Graeme R Polglase, Euan M Wallace, Ryan Hodges, Graham Jenkin, and Suzanne L Miller. Detection and assessment of brain injury in the growth-restricted fetus and neonate. Pediatric research, 82(2):184-193, 2017.

11. Andrew Melbourne, Zach Eaton-Rosen, Enrico De Vita, Alan Bainbridge, Manuel Jorge Cardoso, David Price, Ernest Cady, Giles S. Kendall, Nicola J. Robertson, Neil Marlow, and Sébastien Ourselin. Multi-modal measurement of the myelin-to-axon diameter g-ratio in preterm-born neonates and adult controls. In Lecture Notes in Computer Science (including subseries Lecture Notes in Artificial Intelligence and Lecture Notes in Bioinformatics), 2014.

12. Andrew Melbourne, Zach Eaton-Rosen, Eliza Orasanu, David Price, Alan Bainbridge, M. Jorge Cardoso, Giles S. Kendall, Nicola J. Robertson, Neil Marlow, and Sebastien Ourselin. Longitudinal development in the preterm thalamus and posterior white matter: MRI correlations between diffusion weighted imaging and T2 relaxometry. Human Brain Mapping, 2016.

13. Ana Narberhaus, Emma Lawrence, Matthew P Allin, Muriel Walshe, Philip McGuire, Larry Rifkin, Robin Murray, and Chiara Nosarti. Neural substrates of visual paired associates in young adults with a history of very preterm birth: alterations in fronto-parieto-occipital networks and caudate nucleus. Neuroimage, 47(4):1884-1893, 2009.

14. World Health Organization, The Partnership for Maternal, Newborn \& Child Health, and Save the Children. Born too soon: the global action report on preterm birth, 2012.

15. Robert E Smith, Jacques-Donald Tournier, Fernando Calamante, and Alan Connelly. Sift2: Enabling dense quantitative assessment of brain white matter connectivity using streamlines tractography. Neuroimage, 119:338-351, 2015.

16. J-Donald Tournier, Fernando Calamante, and Alan Connelly. Robust determination of the fibre orientation distribution in diffusion mri: non-negativity constrained super-resolved spherical deconvolution. Neuroimage, 35(4):1459-1472, 2007.

17. Siying Wang, Christian Ledig, Joseph V Hajnal, Serena J Counsell, Julia A Schnabel, and Maria Deprez. Quantitative assessment of myelination patterns in preterm neonates using t2-weighted mri. Scientific reports, 9(1):1-12, 2019. 
18. Hui Zhang, Torben Schneider, Claudia A Wheeler-Kingshott, and Daniel C Alexander. Noddi: practical in vivo neurite orientation dispersion and density imaging of the human brain. Neuroimage, 61(4):1000-1016, 2012. 